\title{
Certain Properties of Some Families of Generalized Starlike Functions with respect to $q$-Calculus
}

\author{
Ben Wongsaijai and Nattakorn Sukantamala \\ Department of Mathematics, Faculty of Science, Chiang Mai University, Chiang Mai 50200, Thailand \\ Correspondence should be addressed to Nattakorn Sukantamala; gaia2556@gmail.com
}

Received 16 July 2016; Accepted 1 September 2016

Academic Editor: Jozef Banas

Copyright (C) 2016 B. Wongsaijai and N. Sukantamala. This is an open access article distributed under the Creative Commons Attribution License, which permits unrestricted use, distribution, and reproduction in any medium, provided the original work is properly cited.

\begin{abstract}
By making use of the concept of $q$-calculus, various types of generalized starlike functions of order $\alpha$ were introduced and studied from different viewpoints. In this paper, we investigate the relation between various former types of $q$-starlike functions of order $\alpha$. We also introduce and study a new subclass of $q$-starlike functions of order $\alpha$. Moreover, we give some properties of those $q$-starlike functions with negative coefficient including the radius of univalency and starlikeness. Some illustrative examples are provided to verify the theoretical results in case of negative coefficient functions class.
\end{abstract}

\section{Introduction and Preliminaries}

The quantum calculus, so called $q$-calculus and $h$-calculus, is the usual calculus without using the notion of limits. The letter $h$ apparently stands for Planck's constant and the letter $q$ obviously stands for quantum. Here, quantum calculus is not the same as quantum physics. Due to the applications in various fields of mathematics and physics, the study of $q$-calculus has been very attractive for many researchers. Jackson $[1,2]$ was the first person in developing a $q$ derivative, also a $q$-integral, in a systematic mean. Afterward on quantum groups, the geometrical interpretation of $q$ analysis has been studied. The relation between $q$-analysis and integrable systems has been recognized. Based on $q$ analogue of beta function, Aral and Gupta [3-5] defined and studied the $q$-analogue of Baskakov Durrmeyer operator. Also, there are some discussions on $q$-Picard and $q$-GaussWeierstrass singular integral operators which are the other important $q$-generalization of complex operators (see [6-8]).

In geometric function theory, there are many applications of $q$-calculus on subclasses of analytic functions, especially subclasses of univalent functions. In [9], Ismail et al. first introduced the class of generalized functions via $q$-calculus. In [10], Raghavendar and Swaminathan have studied some basic properties of q-close-to-convex functions. In [11], Mohammed and Darus studied geometric properties and approximations of these $q$-operators in some subclasses of analytic functions in the disk. By using the convolution of normalized analytic functions and $q$-hypergeometric functions, these $q$-operators have been defined. The inclusive study on applications of $q$-calculus in operator theory could be seen in [12]. Recently, Esra Özkan Uçar [13] studied the coefficient inequality for $q$-closed-to-convex functions with respect to Janowski starlike functions. Here, many newsworthy results related to $q$-calculus and subclasses of analytic functions theory are studied by various authors (see [14-21]).

Let $\mathbb{D}_{r}=\{z \in \mathbb{C}:|z|<r\}$ be the open disk radius $r$ centered at origin and the open unit disk is then defined by $\mathbb{D} \equiv \mathbb{D}_{1}$. We denote $\mathscr{A}$ by the class of functions $f$ in the form

$$
f(z)=z+\sum_{k=2}^{\infty} a_{k} z^{k} \quad(z \in \mathbb{D})
$$

which is analytic in $\mathbb{D}$ and satisfying the usual normalization condition $f(0)=f^{\prime}(0)-1=0$. We denote by $\mathcal{S}$ the subclass of $\mathscr{A}$ consisting of functions, which are univalent on $\mathbb{D}$. A 
function $f \in \mathscr{A}$ is said to be starlike of order $\alpha(0 \leq \alpha<1)$ in $\mathbb{D}$ if $f$ satisfies

$$
\operatorname{Re}\left\{\frac{z f^{\prime}(z)}{f(z)}\right\}>\alpha \quad(z \in \mathbb{D}) .
$$

We denote this class by $\mathcal{S}^{*}(\alpha)$. In particular, we set $\mathcal{S}^{*}(0) \equiv$ $\mathcal{S}^{*}$ for a class of starlike functions on $\mathbb{D}$. Class $\mathcal{S}_{\alpha}^{*}$ is closely related to class $\mathcal{S}^{*}(\alpha)$. A function $f \in \mathscr{A}$ is said to belong to class $\mathcal{S}_{\alpha}^{*}$ if $f$ satisfies

$$
\left|\frac{z f^{\prime}(z)}{f(z)}-1\right|<1-\alpha \quad(z \in \mathbb{D}) .
$$

For the convenience, we provide some basic definitions and concept details of $q$-calculus which are used in this paper. For any fixed complex number $\mu$, a set $A \subset \mathbb{C}$ is called a $\mu$ geometric set if for $z \in A, \mu z \in A$. Let $f$ be a function defined on a $q$-geometric set. Jackson's $q$-derivative and $q$-integral of a function on a subset of $\mathbb{C}$ are, respectively, given by (see Gasper and Rahman [22], pp. 19-22)

$$
\begin{aligned}
D_{q} f(z) & =\frac{f(z)-f(z q)}{z(1-q)}, \quad(z \neq 0, q \neq 0), \\
\int_{0}^{z} f(t) d_{q} t & =z(1-q) \sum_{k=0}^{\infty} q^{k} f\left(z q^{k}\right) .
\end{aligned}
$$

In case $f(z)=z^{n}$, the $q$-derivative and $q$-integral of $f(z)$, where $n$ is a positive integer, are given by

$$
\begin{aligned}
D_{q} z^{n} & =\frac{z^{n}-(z q)^{n}}{(1-q) z}=[n]_{q} z^{n-1} \\
\int_{0}^{z} t^{n} d_{q} t & =z(1-q) \sum_{k=0}^{\infty} q^{k}\left(z q^{k}\right)^{n}=\frac{z^{n+1}}{[n+1]_{q}} .
\end{aligned}
$$

As $q \rightarrow 1^{-}$and $n \in \mathbb{N}$, we have $[n]_{q}=\left(1-q^{n}\right) /(1-q)=$ $1+q+\cdots+q^{n-1} \rightarrow n$.

To generalize the class of starlike functions, it seems that replacing the derivative function $f^{\prime}$, which appears in (2), by the $q$-difference operator $D_{q}$ is an easily way to generalize the class of starlike functions. The definition turned out to be the following.

Definition 1. A function $f \in \mathscr{A}$ is said to belong to class $\mathcal{S}_{q, 1}^{*}(\alpha), 0 \leq \alpha<1$, if

$$
\operatorname{Re}\left\{\frac{z D_{q} f(z)}{f(z)}\right\}>\alpha \quad(z \in \mathbb{D}) .
$$

To put it in words, we call $\mathcal{S}_{q, 1}^{*}(\alpha)$ the class of $q$-starlike functions of order $\alpha$ type 1 .

Now we recall another way to generalize the class of starlike functions proposed by Ismail et al. [9]. In their works, the usual derivative was replaced by the $q$-difference operator $D_{q}$. Moreover, the right-half plane $\{w: \operatorname{Re} w>\alpha\}$ was substituted by an appropriate domain. Later, Agrawal and Sahoo in [14] extended the ideas in [9] to $q$-starlike function of order $\alpha$. Then the definition turned out to be the following.
Definition 2. A function $f \in \mathscr{A}$ is said to belong to class $\mathcal{S}_{q, 2}^{*}(\alpha), 0 \leq \alpha<1$, if

$$
\left|\frac{z\left(D_{q} f(z)\right) / f(z)-\alpha}{1-\alpha}-\frac{1}{1-q}\right|<\frac{1}{1-q} \quad(z \in \mathbb{D}) .
$$

To put it in words, we call $\mathcal{S}_{q, 2}^{*}(\alpha)$ the class of $q$-starlike functions of order $\alpha$ type 2 .

In addition, we now introduce new type of $q$-starlike functions.

Definition 3. A function $f \in \mathscr{A}$ is said to belong to class $\mathcal{S}_{q, 3}^{*}(\alpha), 0 \leq \alpha<1$, if

$$
\left|\frac{z\left(D_{q} f(z)\right)}{f(z)}-1\right|<1-\alpha \quad(z \in \mathbb{D}) .
$$

To put it in words, we call $\mathcal{S}_{q, 3}^{*}(\alpha)$ the class of $q$-starlike functions of order $\alpha$ type 3 .

The main objective of this paper is to characterize in 4 sections. In Section 2, we give some relations between such classes and a sufficient condition via coefficient inequality. In Section 3, we study some properties of those $q$-starlike functions of order $\alpha$ with negative coefficient. Here, some results on the radius of univalent and starlikeness order $\alpha$ on the class of $q$-starlike functions with negative coefficient are obtained. Some illustrative examples of radius of univalent and starlikeness on some functions with negative coefficient are demonstrated in Section 4.

\section{Main Results}

We first show the inclusion theorem via geometric properties of each type of $q$-starlike functions.

Theorem 4. For $0<\alpha<1$, then

$$
\mathcal{S}_{q, 3}^{*}(\alpha) \subset \mathcal{S}_{q, 2}^{*}(\alpha) \subset \mathcal{S}_{q, 1}^{*}(\alpha) .
$$

Proof. Assuming that $f \in \mathcal{S}_{q, 3}^{*}(\alpha)$, by using triangle inequality and (8), we have

$$
\begin{aligned}
& \left|\frac{z\left(D_{q} f(z)\right) / f(z)-\alpha}{1-\alpha}-\frac{1}{1-q}\right| \\
& \quad=\frac{1}{1-\alpha}\left|\frac{z D_{q} f(z)}{f(z)}-\alpha-\frac{1-\alpha}{1-q}\right| \\
& \quad \leq \frac{1}{1-\alpha}\left|\frac{z D_{q} f(z)}{f(z)}-1\right|+\frac{q}{1-q} \leq 1+\frac{q}{1-q} \\
& \quad:=\frac{1}{1-q} .
\end{aligned}
$$




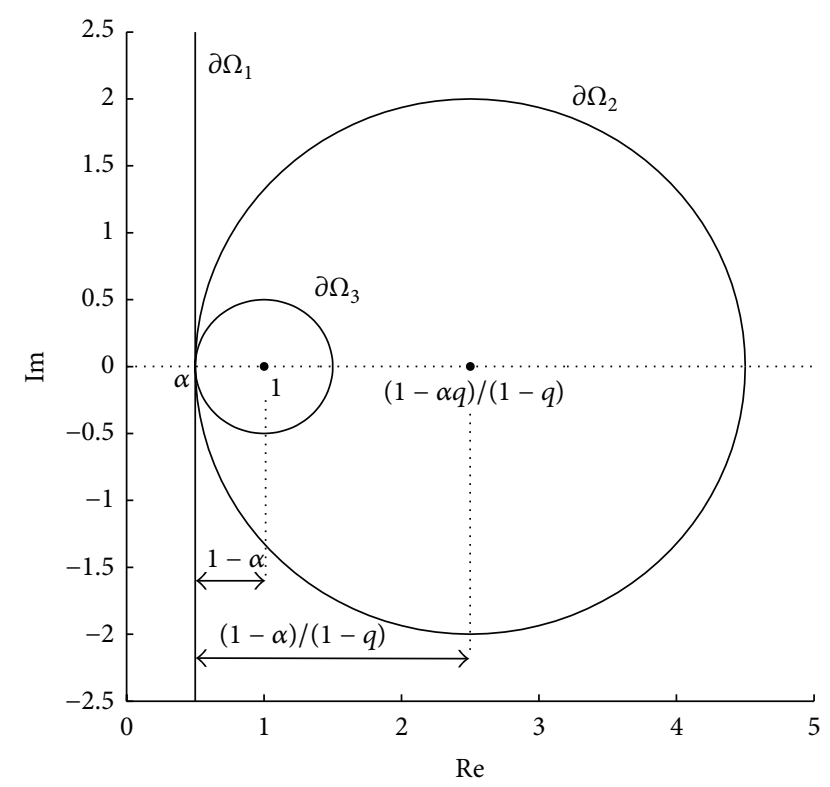

FIGURE 1: Boundary of each domain.

Then $f \in \mathcal{S}_{q, 2}^{*}(\alpha)$; that is, $\mathcal{S}_{q, 3}^{*}(\alpha) \subset \mathcal{S}_{q, 2}^{*}(\alpha)$. Next, we let $f \in \mathcal{S}_{q, 2}^{*}(\alpha)$. Since

$$
f \in \mathcal{S}_{q, 2}^{*}(\alpha) \Longleftrightarrow\left|\frac{z D_{q} f(z)}{f(z)}-\frac{1-\alpha q}{1-q}\right|<\frac{1-\alpha}{1-q},
$$

that is, $z D_{q} f(z) / f(z)$ lies in the circle of radius $(1-\alpha) /(1-q)$ with a center at $(1-\alpha q) /(1-q)$, and we observe that

$$
\frac{1-\alpha q}{1-q}-\frac{1-\alpha}{1-q}=\alpha
$$

which means that $\operatorname{Re}\left\{z D_{q} f(z) / f(z)\right\}>\alpha$, then $f \in \mathcal{S}_{q, 1}^{*}(\alpha)$; that is, $\mathcal{S}_{q, 2}^{*}(\alpha) \subset \mathcal{S}_{q, 1}^{*}(\alpha)$. This completes the proof.

Geometrically, for $f \in \mathcal{S}_{q, k}^{*}(\alpha), k=1,2,3, z D_{q} f(z) / f(z)$ lied in the difference domains:

$$
\begin{aligned}
& \Omega_{1}=\{w \in \mathbb{C}: \operatorname{Re} w>\alpha\}, \\
& \Omega_{2}=\left\{w \in \mathbb{C}:\left|w-\frac{1-\alpha q}{1-\alpha}\right|<\frac{1-\alpha}{1-q}\right\}, \\
& \Omega_{1}=\{w \in \mathbb{C}:|w-1|<1-\alpha\},
\end{aligned}
$$

respectively; see Figure 1.

The next result is directly obtained by using Theorem 4 and the result in [14].

Corollary 5. Classes $\mathcal{S}_{q, 1}^{*}(\alpha), \mathcal{\delta}_{q, 2}^{*}(\alpha)$, and $\mathcal{\delta}_{q, 3}^{*}(\alpha)$ satisfy the following properties:

$$
\begin{aligned}
& \bigcap_{0<q<1} \mathcal{S}_{q, 1}^{*}(\alpha)=\bigcap_{0<q<1} \mathcal{S}_{q, 2}^{*}(\alpha)=\mathcal{S}^{*}(\alpha), \\
& \bigcap_{0<q<1} \mathcal{S}_{q, 1}^{*}(\alpha)=\bigcap_{0<q<1} \mathcal{S}_{q, 3}^{*}(\alpha) \subset \mathcal{S}^{*}(\alpha) .
\end{aligned}
$$

Next, we give a sufficient condition of $\mathcal{S}_{q, 3}^{*}$ via coefficient inequality which guarantees a sufficient condition for $\mathcal{S}_{q, 1}^{*}$ and $\delta_{q, 2}^{*}$.

Theorem 6. If $f \in \mathscr{A}$ satisfies the inequality

$$
\sum_{k=2}^{\infty}\left([k]_{q}-\alpha\right)\left|a_{k}\right| \leq 1-\alpha,
$$

then $f(z)$ is a q-starlike function of order $\alpha$ type 3; that is, $f \in$ $\mathcal{S}_{q, 3}^{*}(\alpha)$.

Proof. Suppose that inequality (15) holds. We obtain

$$
\begin{aligned}
& \left|z D_{q} f(z)-f(z)\right|-(1-\alpha)|f(z)| \\
& \quad=\left|\sum_{k=2}^{\infty}\left([k]_{q}-1\right) a_{k} z^{k}\right|-(1-\alpha)\left|z+\sum_{k=2}^{\infty} a_{k} z^{k}\right| \\
& \quad \leq \sum_{k=2}^{\infty}\left([k]_{q}-1\right)\left|a_{k}\right|-(1-\alpha)\left(1-\sum_{k=2}^{\infty}\left|a_{k}\right|\right) \\
& \quad=\sum_{k=2}^{\infty}\left([k]_{q}-1\right)\left|a_{k}\right|-(1-\alpha) .
\end{aligned}
$$

Then $f \in \mathcal{S}_{q, 3}^{*}(\alpha)$ as desired.

Remark 7. In Theorem 6, if $q \rightarrow 1^{-}$, we obtain Theorem 1 in [23].

\section{Functions with Negative Coefficients}

Now, we introduce new subclasses of $q$-starlike functions with negative coefficients. Let $\mathscr{T}$ be a subset of $\mathscr{A}$ containing negative coefficient functions; that is,

$$
f(z)=z-\sum_{k=2}^{\infty}\left|a_{k}\right| z^{k}
$$

Next, we let

$$
\mathscr{T} \mathcal{S}_{q, k}^{*}(\alpha) \equiv \mathcal{S}_{q, k}^{*}(\alpha) \cap \mathscr{T}, \quad k=1,2,3 .
$$

Theorem 8. For $0<\alpha<1$, then

$$
\mathscr{T} \mathcal{S}_{q, 1}^{*}(\alpha) \equiv \mathscr{T} \mathcal{S}_{q, 2}^{*}(\alpha) \equiv \mathscr{T} \mathcal{S}_{q, 3}^{*}(\alpha) .
$$

Proof. By using Theorem 4, it is sufficient to show that $\mathscr{T} \mathcal{S}_{q, 1}^{*}(\alpha) \subset \mathscr{T} \mathcal{S}_{q, 3}^{*}(\alpha)$. Assuming that $f \in \mathscr{T} \mathcal{S}_{q, 1}^{*}(\alpha)$, we have

$$
\operatorname{Re}\left\{\frac{z D_{q} f(z)}{f(z)}\right\}=\operatorname{Re}\left\{\frac{1-\sum_{k=2}^{\infty}[k]_{q}\left|a_{k}\right| z^{k-1}}{1-\sum_{k=2}^{\infty}\left|a_{k}\right| z^{k-1}}\right\}
$$

$>\alpha$.

Take $z$ on the real axis so that the value of $z D_{q} f(z) / f(z)$ is real. Letting $z$ approach $1^{-}$on the real line, we have

$$
1-\sum_{k=2}^{\infty}[k]_{q}\left|a_{k}\right|>\alpha\left(1-\sum_{k=2}^{\infty}\left|a_{k}\right|\right)
$$


which satisfies (15). Theorem 6 implies the proof of this theorem.

By using the result of Theorem 8 , all types of $q$-starlike functions are exactly the same. For convenience, we introduce a new notation for each class of $q$-starlike functions $\mathscr{T} \mathcal{S}_{q, k}^{*}(\alpha) \equiv \mathscr{T} \mathcal{S}_{q}^{*}(\alpha)$, for $k=1,2$, and 3 .

By using Theorem 6, it is easy to see that function

$$
f_{0}(z)=z-\frac{1-\alpha-\epsilon}{[n]_{q}-\alpha} z^{n} \in \mathscr{T} \mathcal{S}_{q}^{*}(\alpha),
$$

where $0<\epsilon<\left(n(1-\alpha)-[n]_{q}+\alpha\right) / n$ and $[n]_{q}-\alpha<n(1-\alpha-\epsilon)$, but $f_{0}^{\prime}(z)=0$ at $z_{0}=\left[\left([n]_{q}-\alpha\right) / n(1-\alpha-\epsilon)\right]^{1 / n}(\cos (2 k \pi / n)+$ $i \sin (2 k \pi / n)) \in \mathbb{D}$. That is, $f_{0}(z) \notin \mathcal{S}$ and also $f_{0}(z) \notin \mathcal{S}^{*}(\alpha)$. So, it is interesting to study the radius of univalency and starlikeness of class $\mathscr{T} \mathcal{S}_{q}^{*}(\alpha)$.

Lemma 9 is required to prove the radius of univalency and starlikeness. By using the same techniques of Theorem 1 in [24] and Theorem 1 in [25], we can easily prove Lemma 9. So, the proof is omitted.

Lemma 9. If $f \in \mathscr{T}$, then $f$ is univalent on $\mathbb{D}_{r}$ if and only if $f$ is starlike on $\mathbb{D}_{r}$.

Theorem 10. If $f \in \mathscr{T} \mathcal{S}_{q}^{*}(\alpha)$ then $f$ is univalent and starlike in $|z|<r_{0}$, where

$$
r_{0}=\min _{2 \leq k \leq M_{0}}\left[\frac{[k]_{q}-\alpha}{k(1-\alpha)}\right]^{1 /(k-1)}
$$

and $M_{0}$ satisfies $M_{0}>e^{1+|\ln ((1-q)(1-\alpha) /(q+(1-q)(1-\alpha)))|}$.

Proof. To prove this, we need to find $0<r_{0} \leq 1$ such that $\operatorname{Re}\left\{f^{\prime}(z)\right\}>0$ on $\mathbb{D}_{r_{0}}$, where $\mathbb{D}_{r_{0}}=\left\{z \in \mathbb{C}:|z|<r_{0}\right\}$ due to the following formula:

$$
\begin{aligned}
\operatorname{Re} & \left\{\frac{f\left(z_{1}\right)-f\left(z_{2}\right)}{z_{1}-z_{2}}\right\} \\
& =\int_{0}^{1} \operatorname{Re}\left\{f^{\prime}\left(z_{1}+t\left(z_{2}-z_{1}\right)\right)\right\} d t,
\end{aligned}
$$

which implies the univalency. Consider

$$
\begin{aligned}
\operatorname{Re}\left\{f^{\prime}(z)\right\} & =\operatorname{Re}\left\{1-\sum_{k=2}^{\infty} k\left|a_{k}\right| z^{k-1}\right\} \\
& >1-\sum_{k=2}^{\infty} k\left|a_{k}\right| r_{0}^{k-1},
\end{aligned}
$$

for all $|z|<r_{0}$. By the application of Theorem 6 and (25), the inequality $\operatorname{Re}\left\{f^{\prime}(z)\right\}>0$ holds on $\mathbb{D}_{r_{0}}$, where

$$
r_{0}=\inf _{k \geq 2}\left[\frac{[k]_{q}-\alpha}{k(1-\alpha)}\right]^{1 /(k-1)}
$$

Next, we need to find $M_{0} \in \mathbb{N}$ satisfying (23). Let $f$ : $[2, \infty) \rightarrow \mathbb{R}^{+}$be the function defined by

$$
f(x)=\left[\frac{[x]_{q}-\alpha}{x(1-\alpha)}\right]^{1 /(x-1)} .
$$

Differentiating on both sides of (27) logarithmically, we have

$$
\begin{aligned}
& f^{\prime}(x)=\frac{f(x)}{(x-1)^{2}}\left[\ln x-\frac{(x-1) q^{x} \ln q}{q+A-q^{x}}\right. \\
& \left.\quad+\ln \frac{A}{q+A-q^{x}}-\frac{x-1}{x}\right],
\end{aligned}
$$

where $A=(1-q)(1-\alpha)$. It is easy to see that the second term of (28) is positive. Since

$$
\begin{aligned}
\sup _{x \geq 2}\left|\ln \frac{A}{q+A-q^{x}}\right| & =\left|\ln \frac{A}{q+A}\right|, \\
\sup _{x \geq 2} \frac{x-1}{x} & =1,
\end{aligned}
$$

then the third and the last term in (28) can be dominated by $\ln x$ when $x$ is sufficiently large. That implies that $f$ is an increasing function on $\left[M_{0}, \infty\right]$, where $M_{0}>e^{1+|\ln (A /(q+A))|}$. Therefore, the radius of univalency can be defined by

$$
\begin{aligned}
r_{0} & =\inf _{k \geq 2}\left[\frac{[k]_{q}-\alpha}{k(1-\alpha)}\right]^{1 /(k-1)} \\
& =\min _{2 \leq k \leq M_{0}}\left[\frac{[k]_{q}-\alpha}{k(1-\alpha)}\right]^{1 /(k-1)} .
\end{aligned}
$$

Finally, we complete the proof of this theorem by applying Lemma 9 to obtain the radius of starlikeness.

Theorem 11 guarantees the radius of starlike function of order $\alpha$.

Theorem 11. If $f \in \mathscr{T} \mathcal{S}_{q}^{*}(\alpha)$ then $f$ is starlike order $\alpha$ in $|z|<$ $r_{1}$, where

$$
r_{1}=\min _{2 \leq k \leq M_{1}}\left[\frac{[k]_{q}-\alpha}{k-\alpha}\right]^{1 /(k-1)},
$$

and $M_{1}$ satisfies $M_{1}>e^{1+|\ln ((1-q) /(1-\alpha(1-q)))|}$.

Proof. We have to show that $\left|z f^{\prime}(z) / z-1\right|<1-\alpha$. That is,

$$
\begin{aligned}
\left|\frac{z f^{\prime}(z)}{f(z)}-1\right| & =\left|\frac{\sum_{k=2}^{\infty}(k-1)\left|a_{k}\right| z^{k-1}}{1-\sum_{k=2}^{\infty}\left|a_{k}\right| z^{k-1}}\right| \\
& \leq \frac{\sum_{k=2}^{\infty}(k-1)\left|a_{k}\right||z|^{k-1}}{1-\sum_{k=2}^{\infty}\left|a_{k}\right||z|^{k-1}} \leq 1-\alpha .
\end{aligned}
$$

Hence, (32) is true if

$$
\sum_{k=2}^{\infty}(k-\alpha)\left|a_{k}\right||z|^{k-1} \leq 1-\alpha .
$$



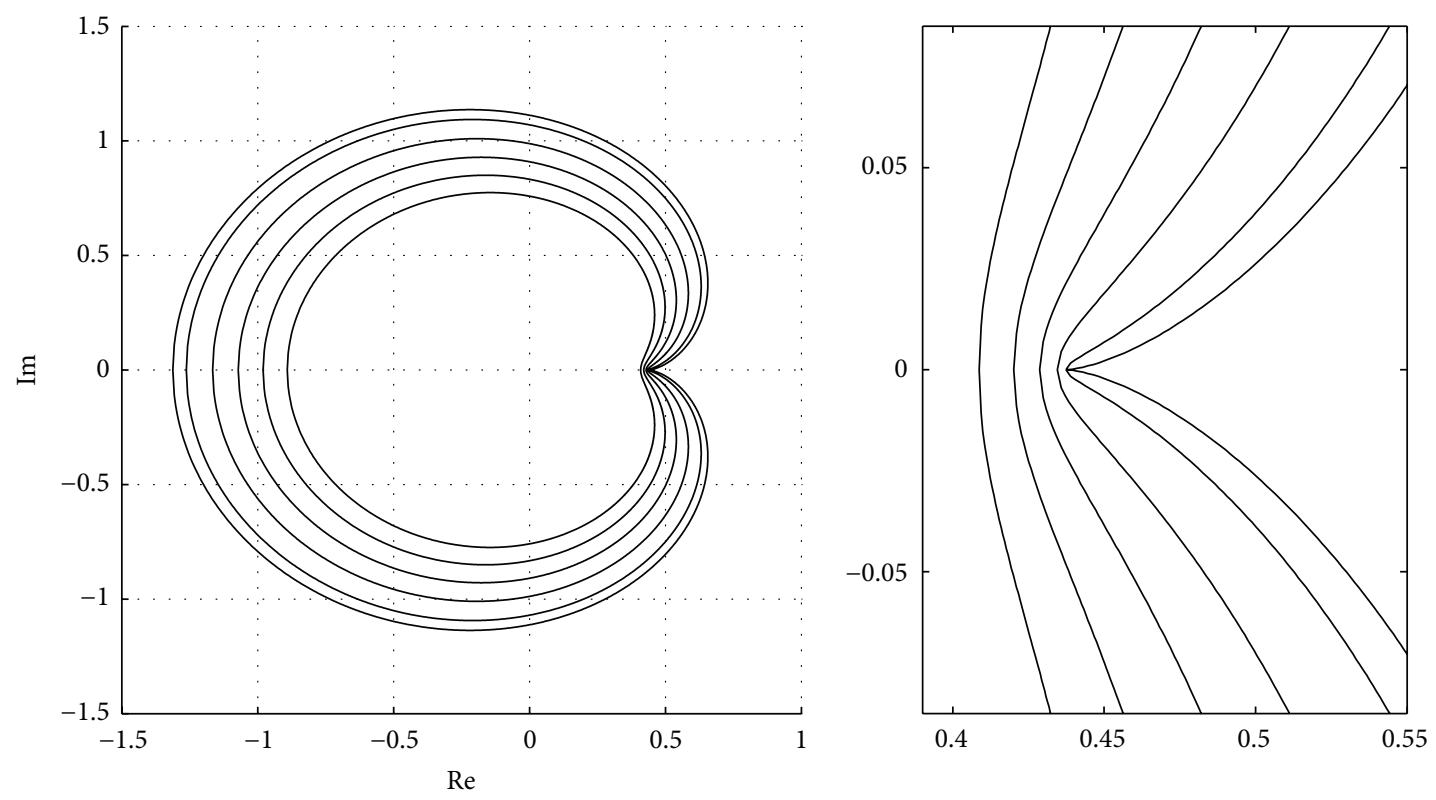

(a)
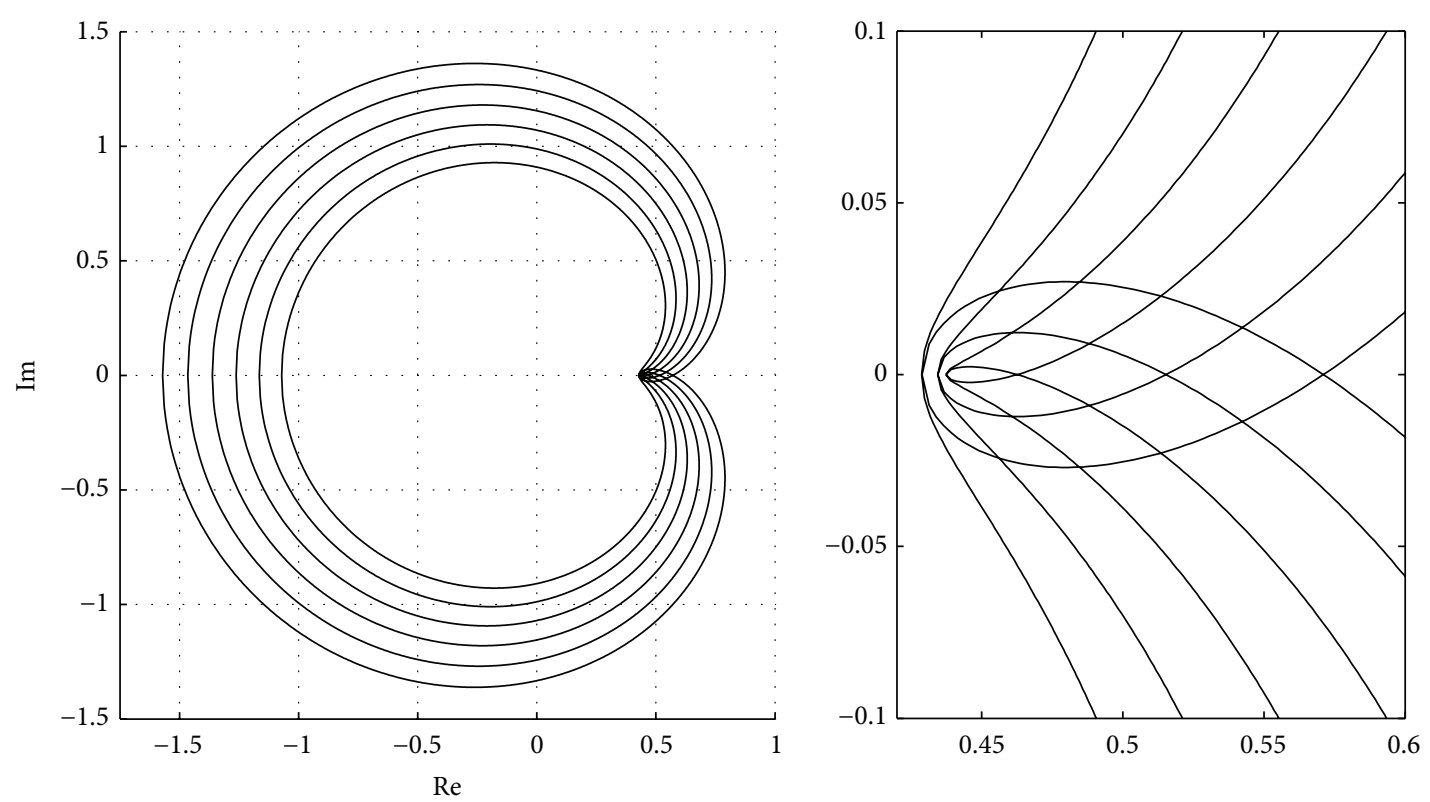

(b)

FIgURE 2: The image of $\partial \mathbb{D}_{r}$ with maximum circumferences $r=0.875$ (a) and $r=1$ (b) on the polynomial $f_{0}(z)$ defined in (36).

By an application of Theorem 6, the above inequality holds on $\mathbb{D}_{r_{1}}$, where

$$
r_{1}=\inf _{k \geq 2}\left[\frac{[k]_{q}-\alpha}{k-\alpha}\right]^{1 /(k-1)}
$$

Finally, by using the same technique of Theorem 10, we obtain that function $f(x)=\left[\left([k]_{q}-\alpha\right) /(k-\alpha)\right]^{1 /(k-1)}$ is an increasing function on $\left[M_{1}, \infty\right)$, where $M_{1}$ satisfies $M_{1}>$ $e^{1+|\ln ((1-q) /(1-\alpha(1-q)))|}$. This completes the proof.

\section{Examples and Applications}

In this section, we give some examples to verify the radius of univalency and starlikeness obtained by Theorems 10 and 11.

Example 1. Consider class $\mathscr{T} \mathcal{S}_{q}^{*}$ with $q=0.75$.

By Theorem 10, we obtain the radius of univalency of class $\mathscr{T} \mathcal{S}_{q}^{*}$ given by

$$
r_{0}=\min _{2 \leq k \leq e^{\lceil 1+|\ln 0.25|\rceil}}\left[\frac{[k]_{0.75}}{k}\right]^{1 /(k-1)}
$$



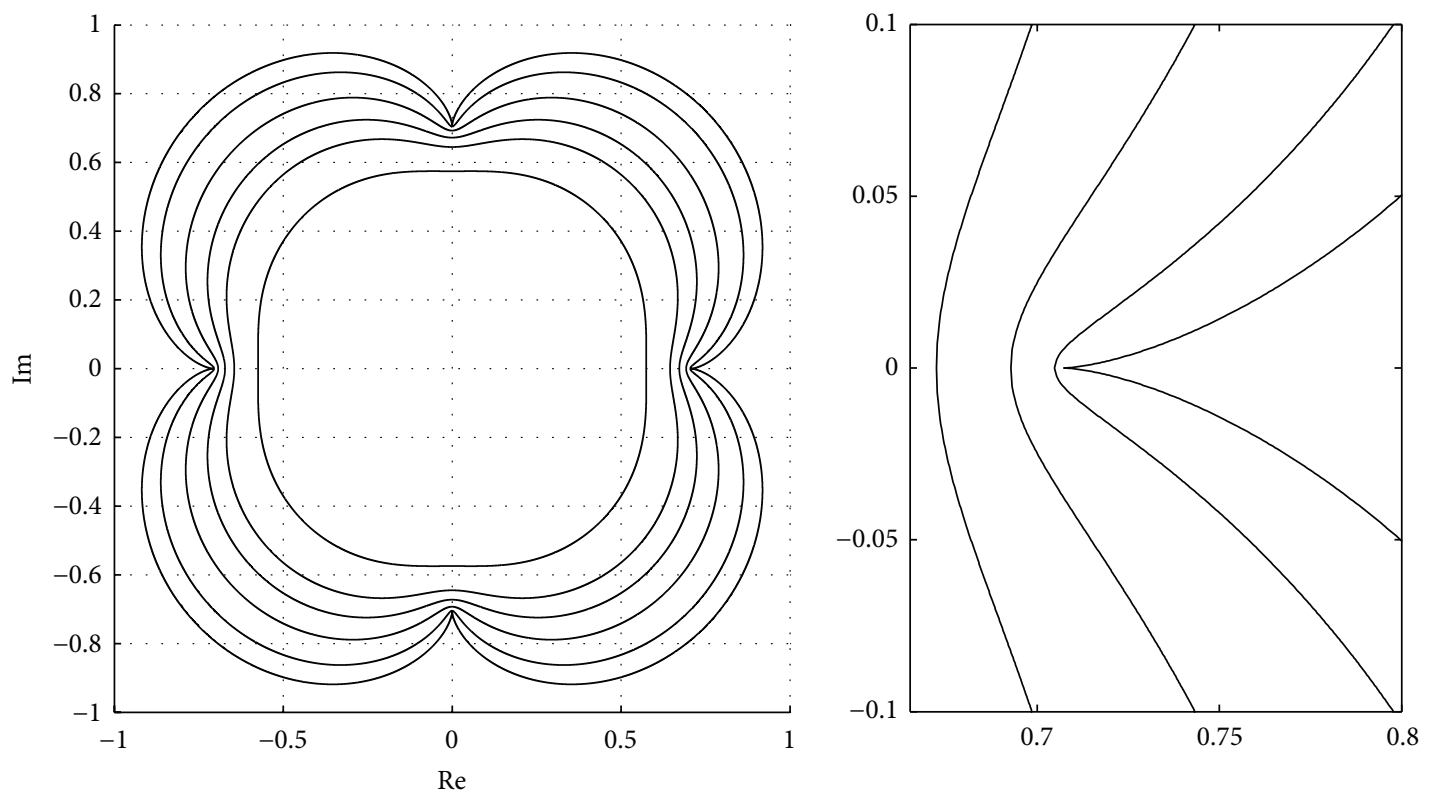

FIgURE 3: The image of $\partial \mathbb{D}_{r}$ with maximum circumferences $r=0.884$ under the polynomial $f_{0}(z)$ defined in (37).

$$
=\min _{2 \leq k \leq 11}\left[\frac{[k]_{0.75}}{k}\right]^{1 /(k-1)}=0.875 \text {. }
$$

Now, we consider the sharpness example function $f_{0}(z)$ defined in (22) with $n=2$ and $\epsilon=0.001$; that is,

$$
f_{0}(z)=z-\frac{0.999}{1.75} z^{2}
$$

Obviously, $f_{z}(z)$ is locally univalent on $\mathbb{D}_{0.875}$ because $f^{\prime}\left(z_{0}\right)=0$ at $z_{0} \approx 0.87587 \ldots$ outside the open disk $\mathbb{D}_{0.875}$. By applying Theorem 10 , function $f_{0}(z)$ is univalent on $\mathbb{D}_{0.875}$. Moreover, Figure 2 shows the image of $\partial \mathbb{D}_{r}$ with maximum circumferences $r=0.875$ and $r=1$. Figure 2(a) demonstrates that function $f_{0}(z)$ is a univalent and starlike function on $\mathbb{D}_{0.875}$. On the other hand, $f_{0}(z)$ is not a univalent on $\mathbb{D}$ (see Figure 2(b)).

Another example is in case $n=5$ with $\epsilon=0.001$; that is,

$$
f_{0}(z)=z-\frac{0.999}{[5]_{0.75}} z^{5}
$$

We see that $f$ is not locally univalent at $z_{0}=$ $[5]_{0.75} / 4.995^{1 / 4}(\cos (k \pi / 2)+i \sin (k \pi / 2))$, for $k=0,1,2,3$ with $\left|z_{0}\right|=0.88403 \ldots$. Figure 3 shows that function $f_{0}$ defined in (37) is univalent and starlike on $D_{0.88403}$ which contains the open disk $\mathbb{D}_{0.875}$ from Theorem 10. That is, the example shows that radius $r_{0}$ in Theorem 10 is only the sufficient condition for univalency and starlikeness but it is not necessary condition due to function $f_{0}(z)$ defined in (37).

The next example is the class of $q$-starlike functions of order $\alpha$.

Example 2. Consider class $\mathscr{T} \mathcal{S}_{q}^{*}(\alpha)$ with $q=0.75$.
In this example, we also set $q=0.75$. For $\alpha=0.5$, by Theorem 10, we obtain the radius of univalency of class $\mathscr{T} \mathcal{S}_{q}^{*}(0.5)$ given by

$$
\begin{aligned}
r_{0} & =\min _{2 \leq k \leq e^{[1+\mid \ln ((1-q))(1-\alpha) /(q+(1-q))(1-\alpha))) !]}}\left[\frac{[k]_{0.75}}{k}\right]^{1 /(k-1)} \\
& =\min _{2 \leq k \leq 19}\left[\frac{[k]_{0.75}}{k}\right]^{1 /(k-1)} \approx 0.94554 .
\end{aligned}
$$

However, function $f_{0}(z)$ defined in (22) with $n=2$ and $\epsilon=$ 0.001 , that is,

$$
f_{0}(z)=z-\frac{0.499}{1.25} z^{2}
$$

is locally univalent on $\mathbb{D}_{1.2525}$ which contains the open disk $\mathbb{D}_{0.925}$. Then it seems that function $f_{0}(z)$ is univalent and starlike on $\mathbb{D}$ as demonstrated by Figure 4(a). Also function $f_{0}(z)$ defined in (22) with $n=5$ and $\epsilon=0.001$, that is,

$$
f_{0}(z)=z-\frac{0.499}{[5]_{0.75}-0.5} z^{5}
$$

is locally univalent on $\mathbb{D}_{1.0055}$ and it seems that function $f_{0}(z)$ is univalent and starlike on $\mathbb{D}$ as demonstrated by Figure 4(b).

\section{Competing Interests}

The authors declare that they have no conflict of interests.

\section{Acknowledgments}

This research was supported by Department of Mathematics, Faculty of Science, Chiang Mai University. 

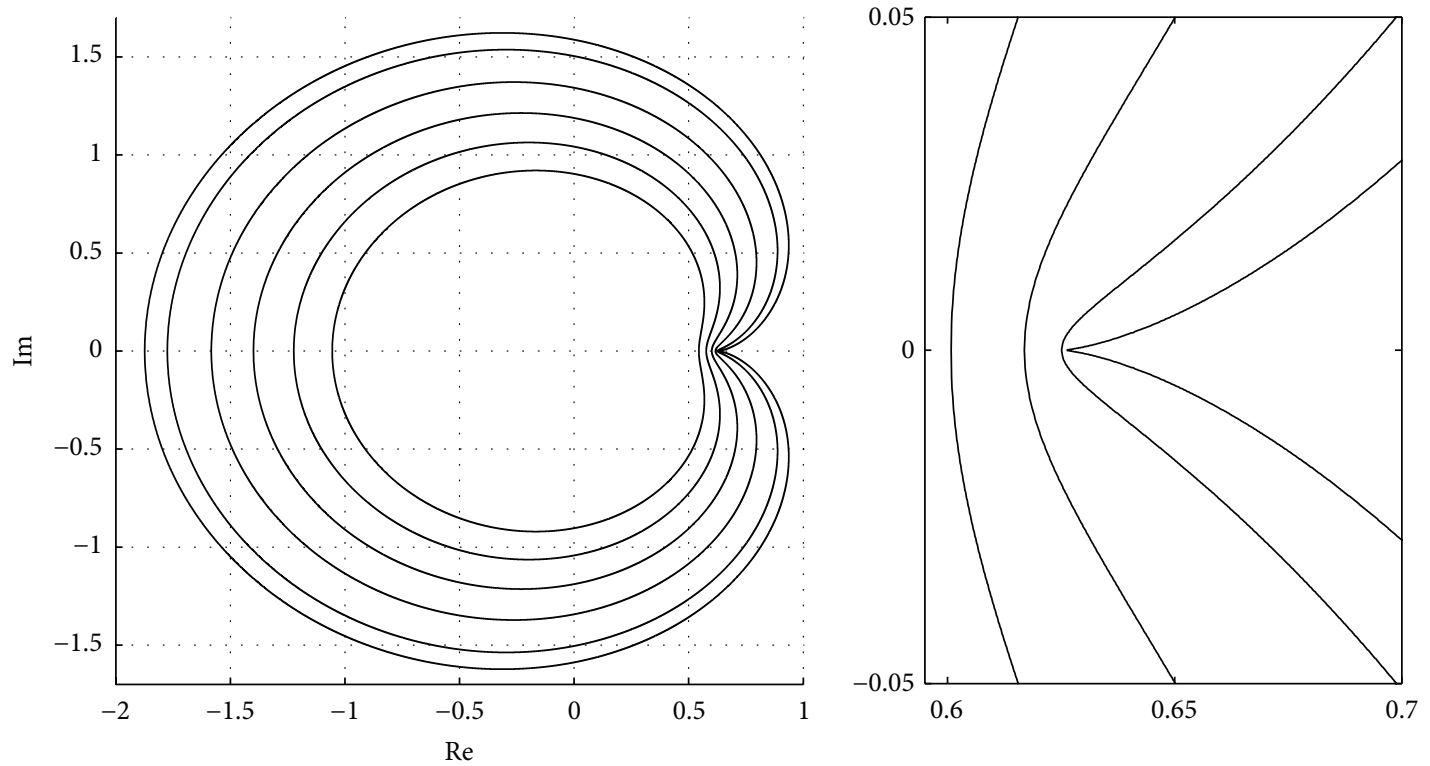

(a)
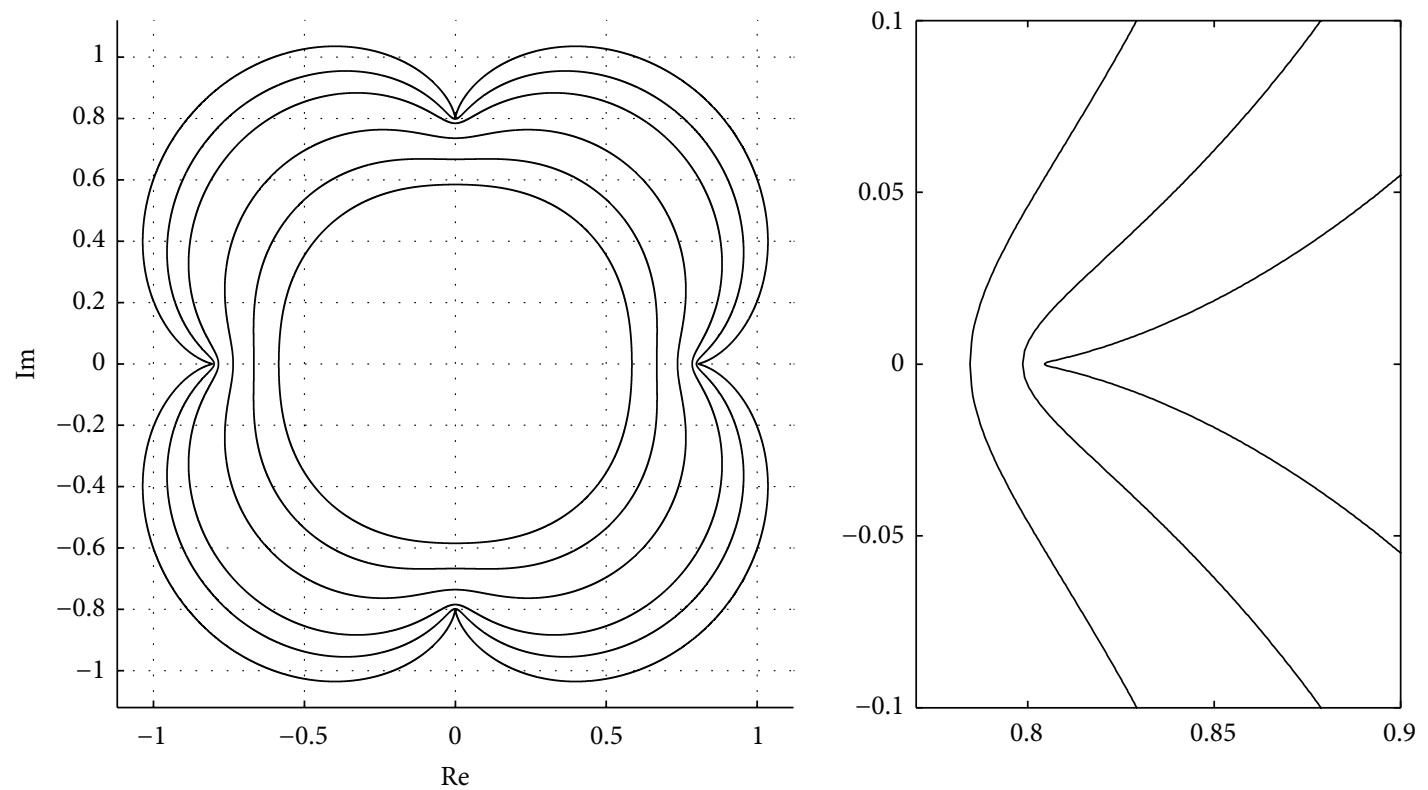

(b)

Figure 4: The image of $\partial \mathbb{D}_{r}$ with maximum circumferences $r=1.2525$ (a) and $r=1.005$ (b) on the polynomial $f_{0}(z)$ defined in (39) and (40), respectively.

\section{References}

[1] F. H. Jackson, "On q-functions and a certain difference operator," Transactions of the Royal Society of Edinburgh, vol. 46, no. 2, pp. 253-281, 1908.

[2] F. H. Jackson, "On q-definite integrals," Quarterly Journal of Pure and Applied Mathematics, vol. 41, pp. 193-203, 1910.

[3] A. Aral and V. Gupta, "On $q$-Baskakov type operators," Demonstratio Mathematica, vol. 42, no. 1, pp. 109-122, 2009.
[4] A. Aral and V. Gupta, "On the Durrmeyer type modification of the q-Baskakov type operators," Nonlinear Analysis: Theory, Methods \& Applications, vol. 72, no. 3-4, pp. 1171-1180, 2010.

[5] A. Aral and V. Gupta, "Generalized q-Baskakov operators," Mathematica Slovaca, vol. 61, no. 4, pp. 619-634, 2011.

[6] G. A. Anastassiou and S. G. Gal, "Geometric and approximation properties of some singular integrals in the unit disk," Journal of Inequalities and Applications, vol. 2006, Article ID 17231, 19 pages, 2006. 
[7] G. A. Anastassiou and S. G. Gal, "Geometric and approximation properties of generalized singular integrals in the unit disk," Journal of the Korean Mathematical Society, vol. 43, no. 2, pp. 425-443, 2006.

[8] A. Aral, "On the generalized Picard and Gauss Weierstrass singular integrals," Journal of Computational Analysis and Applications, vol. 8, no. 3, pp. 249-261, 2006.

[9] M. E. H. Ismail, E. Merkes, and D. Styer, "A generalization of starlike functions," Complex Variables, vol. 14, no. 1-4, pp. 7784, 1990.

[10] K. Raghavendar and A. Swaminathan, "Close-to-convexity of basic hypergeometric functions using their Taylor coefficients," Journal of Mathematics and Applications, vol. 35, pp. 111-125, 2012.

[11] A. Mohammed and M. Darus, "A generalized operator involving the q-hypergeometric function," Matematichki Vesnik, vol. 65, no. 4, pp. 454-465, 2013.

[12] A. Aral, V. Gupta, and R. P. Agarwal, Applications of q-Calculus in Operator Theory, Springer, New York, NY, USA, 2013.

[13] H. Esra Özkan Uçar, "Coefficient inequality for $q$-starlike functions," Applied Mathematics and Computation, vol. 276, pp. 122-126, 2016.

[14] S. Agrawal and S. K. Sahoo, "A generalization of starlike functions of order alpha," https://arxiv.org/abs/1404.3988.

[15] S. K. Sahoo and N. L. Sharma, "On a generalization of close-toconvex functions," http://arxiv.org/abs/1404.3268v1.

[16] H. Al dweby and M. Darus, "On harmonic meromorphic functions associated with basic hypergeometric functions," The Scientific World Journal, vol. 2013, Article ID 164287, 7 pages, 2013.

[17] H. Aldweby and M. Darus, "Some subordination results on $q$-analogue of Ruscheweyh differential operator," Abstract and Applied Analysis, vol. 2014, Article ID 958563, 6 pages, 2014.

[18] G. Murugusundaramoorthy, C. Selvaraj, and O. S. Babu, "Subclasses of starlike functions associated with fractional $q$-calculus operators," Journal of Complex Analysis, vol. 2013, Article ID 572718, 8 pages, 2013.

[19] K. A. Selvakumaran, S. D. Purohit, and A. Secer, "Majorization for a class of analytic functions defined by q-differentiation," Mathematical Problems in Engineering, vol. 2014, Article ID 653917, 5 pages, 2014.

[20] K. A. Selvakumaran, S. D. Purohit, A. Secer, and M. Bayram, "Convexity of certain $q$-integral operators of $p$-valent functions," Abstract and Applied Analysis, vol. 2014, Article ID 925902, 7 pages, 2014.

[21] B. Wongsaijai and N. Sukantamala, "Applications of fractional $q$-calculus to certain subclass of analytic $p$-valent functions with negative coefficient," Abstract and Applied Analysis, vol. 2015, Article ID 273236, 12 pages, 2015.

[22] G. Gasper and M. Rahman, Basic Hypergeometric Series, Cambridge University Press, Cambridge, UK, 1990.

[23] H. Silverman, "Univalent functions with negative coefficients," Proceedings of the American Mathematical Society, vol. 51, pp. 109-116, 1975.

[24] Q. Deng, "On univalent functions with negative coefficients," Applied Mathematics and Computation, vol. 189, no. 2, pp. 16751682, 2007.

[25] A. W. Goodman, "Univalent functions and nonanalytic curves," Proceedings of the American Mathematical Society, vol. 8, pp. 598-601, 1957. 


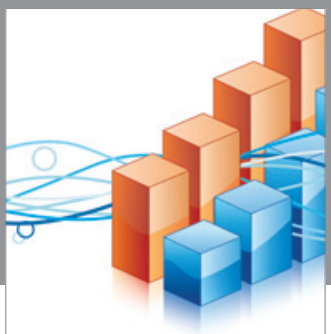

Advances in

Operations Research

vatem alat4

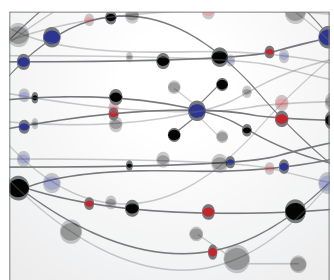

\section{The Scientific} World Journal
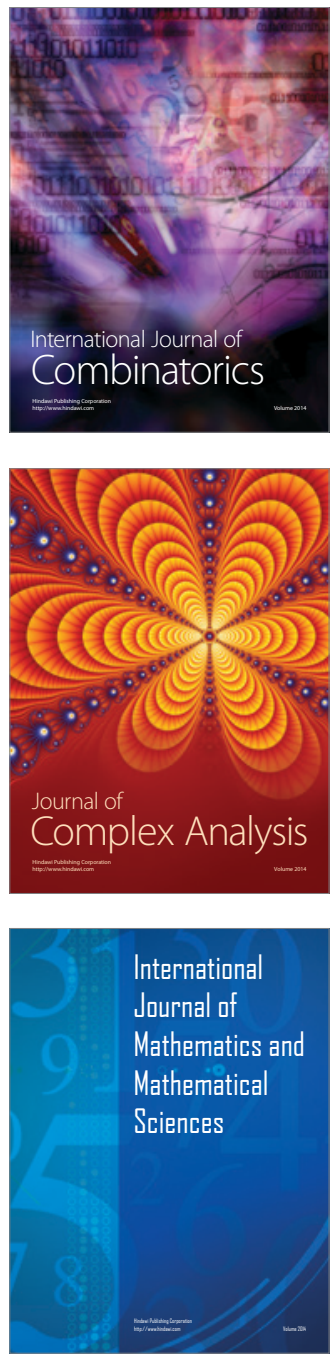
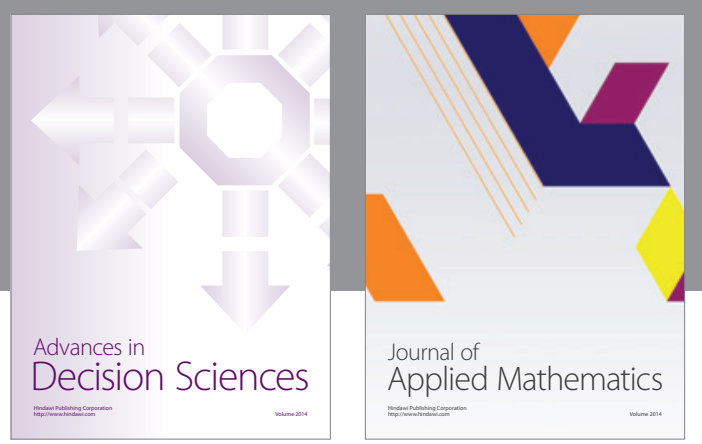

Algebra

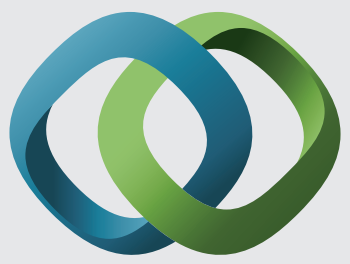

\section{Hindawi}

Submit your manuscripts at

http://www.hindawi.com
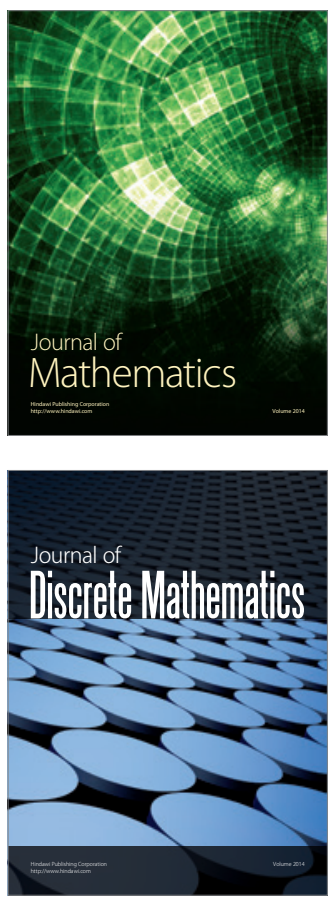

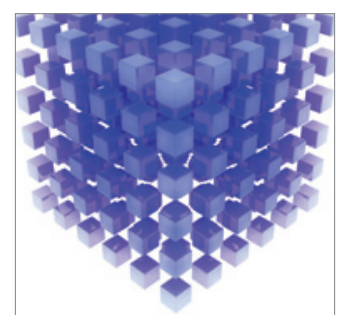

Mathematical Problems in Engineering
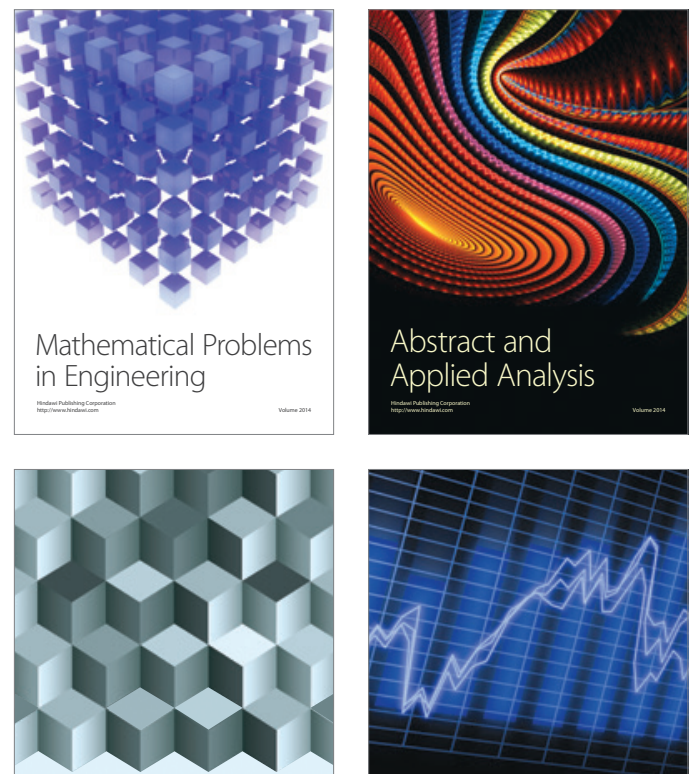

Journal of

Function Spaces

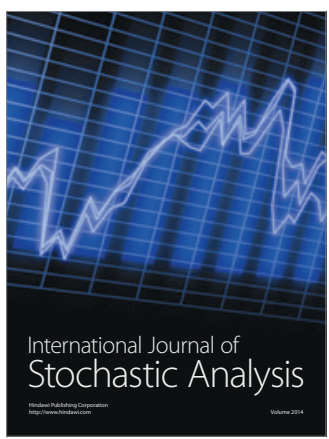

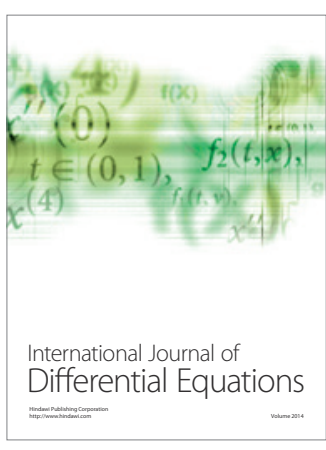
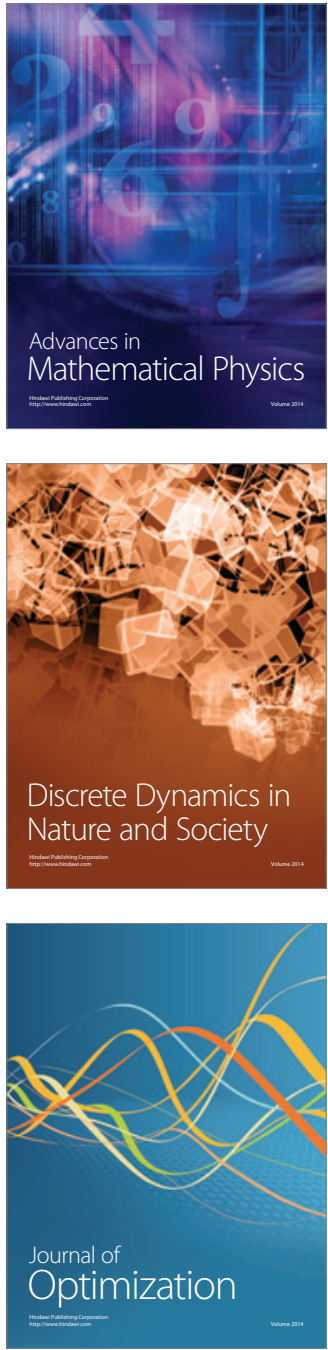